\title{
Impact of Intraendometrial Vascularity on Implantation Rates in Frozen Blastocyst Transfer
}

\author{
Jalpa Shah ${ }^{1}$, Chaitanya Nagori $^{2}$, Sonal Panchal ${ }^{3}$, Manish Thaker ${ }^{4}$
}

\begin{abstract}
Introduction: Implantation remains the main limiting factor in the success of in vitro fertilization treatment, despite major advances made in this field. This study was done to evaluate whether intraendometrial vascularity affects the outcome in cases of Frozen embryo transfer (FET).

Materials and methods: In total, 226 patients undergoing frozen embryo transfer out of the total 233 patients were recruited for the study. Endometrial preparation was done by artificial cycle protocol in all the patients with estradiol valerate. Endometrium is evaluated for its thickness and its morphology as seen on B-mode ultrasound. Endometrial vascularity is evaluated by power Doppler (PD) and pulse-wave Doppler. Post-embryo transfer of two Grade 1 frozen-thawed blastocyst, judicial luteal support was given. Beta human chorionic gonadotropin ( $\beta$-hCG) was checked after 14 days for confirmation of pregnancy in all the patients. In all $\beta$-hCG positive cases, ultrasound was done after 10 days. All the patients with pregnancy were followed up by ultrasound for the entire first trimester and miscarriages were documented.

Results: Patients showed higher positive pregnancy rate and lesser miscarriage rate with vascular penetration in zone 3 and zone 4 of the endometrium. Conception rate and miscarriage rate did not vary significantly with change in endometrial thickness beyond $7 \mathrm{~mm}$ in Frozen embryo transfer cycles.

Keywords: Blastocyst, Conception rates, Endometrial vascularity, Frozen embryo transfer, Miscarriage rates.

Donald School Journal of Ultrasound in Obstetrics and Gynecology (2021): 10.5005/jp-journals-10009-1823
\end{abstract}

\section{INTRODUCTION}

Successful implantation is a result of a positive dialogue between the endometrium and embryo. ${ }^{1}$ For a very short time during the menstrual cycle, the endometrium allows for the implantation of gestational sac and this is referred to as endometrial receptivity. ${ }^{2}$ There are many variables of successful implantation and those related to embryo are difficult to study inspite of all advances in technology. Molecular changes play a major role in endometrial receptivity and are also controlled by hormonal changes. These reflect as changes in endometrial morphology and vascularity.

Transvaginal ultrasonography is universally accepted and most extensively used noninvasive tool to assess the endometrial receptivity during assisted reproductive treatment. ${ }^{3}$ Ultrasound is easily repeatable, yet highly reproducible, patient friendly and yet accurate modality for endometrial receptivity. ${ }^{4}$ Assessment of blood flow as a functional dimension of endometrial receptivity along with endometrial thickness, has important role in the prediction of pregnancy outcomes FET (frozen embryo transfer) cycles. ${ }^{5-7}$ This study is aimed to assess the impact of endometrial morphology, thickness and endometrial vascularity in pregnancy outcomes in FET cycles.

\section{Materials and Methods}

\section{Study Design}

This is retrospective cross-sectional study. Total 235 patients undergoing frozen embryo transfers (FET) in our centre from December 2019 to December 2020 were recruited for the study. The study was approved by the institutional ethical committee.

\section{Inclusion Criteria}

- Females between 20 and 40 years of age.

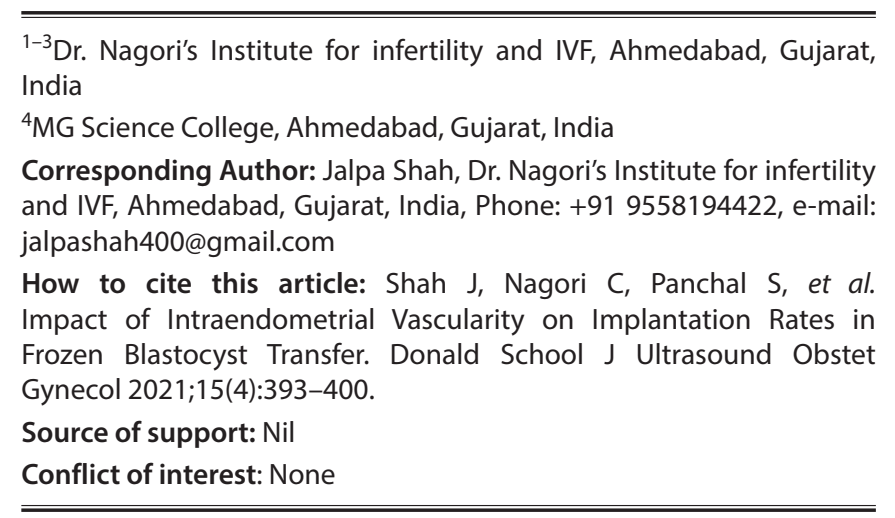

- Females with BMI-22-29.9.

- Patients with normal uterus (no Mullerian anomalies).

- Patients with mild adenomyosis, that allows endometrium to grow to at least $6 \mathrm{~mm}$.

- Patients with no endometrial pathology like polyp, subendometrial fibroid, acute or chronic endometritis.

- Patients with mild/moderate endometriosis.

\section{Exclusion Criteria}

- Females under 20 years and above 40 years of age.

- Females with BMI more than 22 and less than 30.

- Patients with severe endometriosis.

- Patients with Mullerian anomalies.

- Patients with endometrial polyps, submucous fibroids or endometriosis.

- Patients with cervical pathologies that restrict the passage of embryo transfer cannula.

- Patients with uterine artery $\mathrm{PI}>3.2 .^{12}$

\footnotetext{
() The Author(s). 2021 Open Access This article is distributed under the terms of the Creative Commons Attribution 4.0 International License (https://creativecommons. org/licenses/by-nc/4.0/), which permits unrestricted use, distribution, and non-commercial reproduction in any medium, provided you give appropriate credit to the original author(s) and the source, provide a link to the Creative Commons license, and indicate if changes were made. The Creative Commons Public Domain Dedication waiver (http://creativecommons.org/publicdomain/zero/1.0/) applies to the data made available in this article, unless otherwise stated.
} 


\section{Method}

For all the patients, a pilot transvaginal ultrasonography was performed with transvaginal volume probe of $5-9 \mathrm{MHz}$, on Voluson E10 BT20 (GE Medical Systems Kretztechnik GmbH, Zipf, Austria) during the preovulatory phase of the previous cycle. Systematic and detailed scan of the pelvic organs-uterus, ovaries, and fallopian tubes was done to exclude any abnormalities for each patient.

Out of total of 233 patients who underwent FET during the study period, seven patients were excluded from the study. Of these one patient had endometrial polyp, two patients had intramural fibroid, larger than $4 \mathrm{~cm}$ in diameter, one patient had subendometrial fibroid, one patient was at 42 years of age and two patients had B.M.I. of more than 30 . Total 226 patients were then taken forward for the study.

Estradiol valerate $2 \mathrm{mg}$ was given thrice a day orally to these patients, from day 2 of the treatment cycle for endometrial preparation along with aspirin $75 \mathrm{mg}$ given orally once daily. The endometrium was assessed for its morphology and thickness on day 8-9 of the cycle.

B-mode ultrasound was used to assess the endometrial thickness and morphology. Endometrium was assessed in the mid-sagittal plane of the uterus. B-mode image is optimized

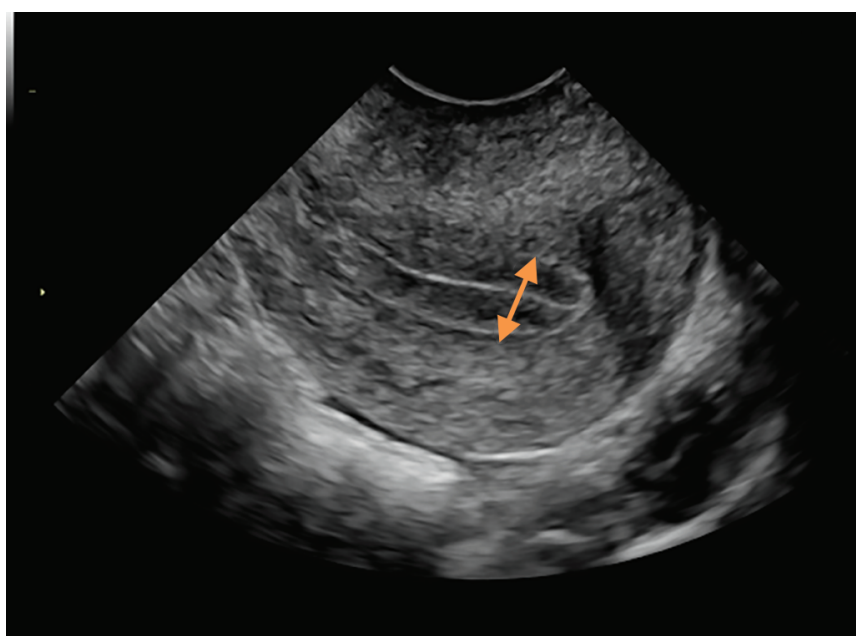

Fig. 1: B-mode image of the mid-sagittal plane of the uterus showing endometrial thickness measurement by bringing the uterus in the centre of the image so that it covers $2 / 3$ rd of the screen, adjusting the angle, depth, and focal zone. Endometrial thickness was measured from the outer margin of the anterior hyperechoic line of the endometrium to the outer margin of posterior hyperechoic line of the endometrium at the broadest part of the endometrium, excluding the junctional zone; perpendicular to the central endometrial line of the endometrium (Fig. 1). Depending on the thickness of the endometrium, a repeat visit is planned when the endometrium is likely to grow till the thickness of at least $6 \mathrm{~mm} .{ }^{8}$ This can be decided on the basis of the fact that endometrium grows at a rate of $1-2 \mathrm{~mm}$ per day. ${ }^{8}$

Morphologically the multi-layered endometrium ${ }^{9}$ is graded as (Fig. 2):

Grade A: Triple line endometrium with echogenicities in intervening area not more than that of anterior myometrial echogenicity.

Grade B: Multilayered or triple line endometrium with hypoechoic intervening area.

Grade C: Homogeneous isoechoiec endometrium.

Endometrial vascularity was assessed with HD flow power Doppler. For HD flow power Doppler, PRF is adjusted at 0.3 , wall filter at low 1 and volume gate size of $2 \mathrm{~mm}$. The Doppler box is placed on the mid-sagittal plane of the endometrium, large enough to include the entire endometrium.

Vascularity is graded according to Applebaum ${ }^{10}$ as (Fig. 3):

Zone 1: When blood vessels reach the hypoechoic endometrial-myometrial junction.

Zone 2: When blood vessels reach the outer hyperechoic line of endometrium.

Zone 3: When blood vessels reach the intervening hypoechoic area between the two hyperechoic lines.

Zone 4: When blood vessels reached the central echogenic line.

At least $5 \mathrm{~mm}^{2}$ area of a particular zone must show vascularity to designate that zone is adequately vascularized ${ }^{11}$ (Fig. 4). Pulse-wave Doppler is used to assess the flow in these spiral vessels, supplying the endometrium. At least 4-5 good waveforms are obtained; each demonstrating the maximum Doppler shift.

The resistance index (RI) was calculated on three consecutive uniform waveforms. The pulse Doppler of spiral vessels with RI 0.49-0.59 was considered a favorable prognostic factor for conception. $^{14}$
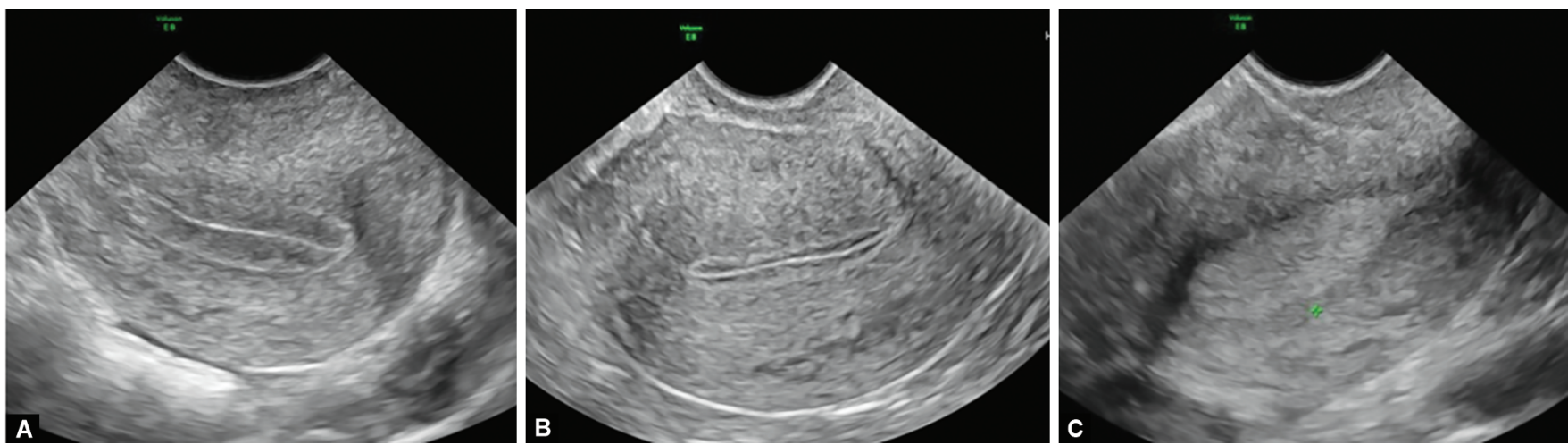

Figs $2 A$ to C: (A) B-mode ultrasound image of mid-sagittal plane of the uterus showing grade A morphology with thick multilayered endometrium and smooth central line. The intervening area between the central line and outer lines show echogenicities similar to that of anterior myometrium; (B) B-mode ultrasound image of mid-sagittal plane of the uterus showing grade B morphology with thick multilayered endometrium and smooth central line. The intervening area between the central line and outer lines is hypoechoic; (C) B-mode ultrasound image of mid-sagittal plane of the uterus showing grade $\mathrm{C}$ morphology with thick but homogeneous endometrium, isoechoic to the myometrium 

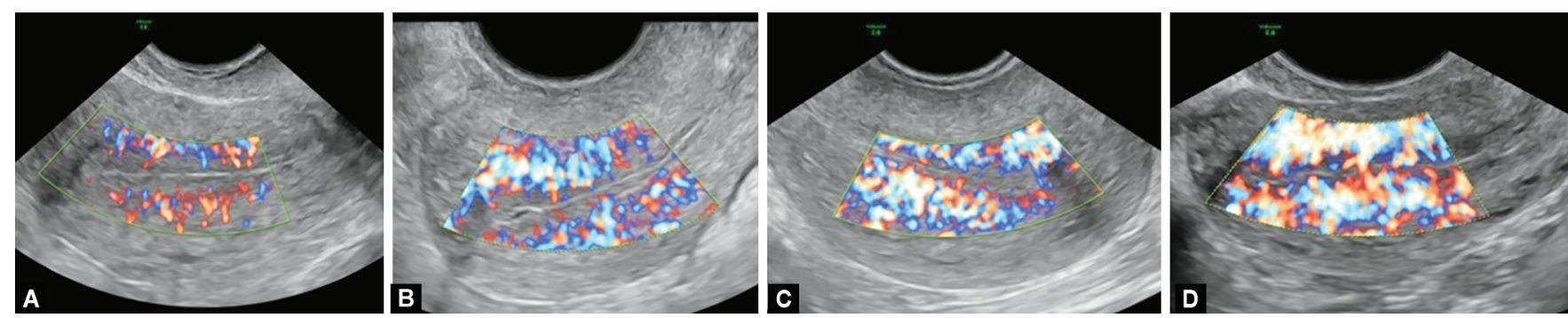

Figs $3 \mathrm{~A}$ to $\mathrm{D}$ : (A) Mid-sagittal plane of the uterus with high definition power Doppler showing blood vessels only up to zone 1 (till endometrio-myometrial junction); (B) Mid-sagittal plane of the uterus with HD power Doppler showing blood vessels up to zone 2 (till outer hyperechoeic lines of the endometrium); (C) Mid-sagittal plane of the uterus with HD power Doppler showing blood vessels up to zone 3 (till the intervening area between the outer hyperechoeic lines of endometrium); (D) Mid-sagittal plane of the uterus with HD power Doppler showing blood vessels up to zone 4 (touching the central line)

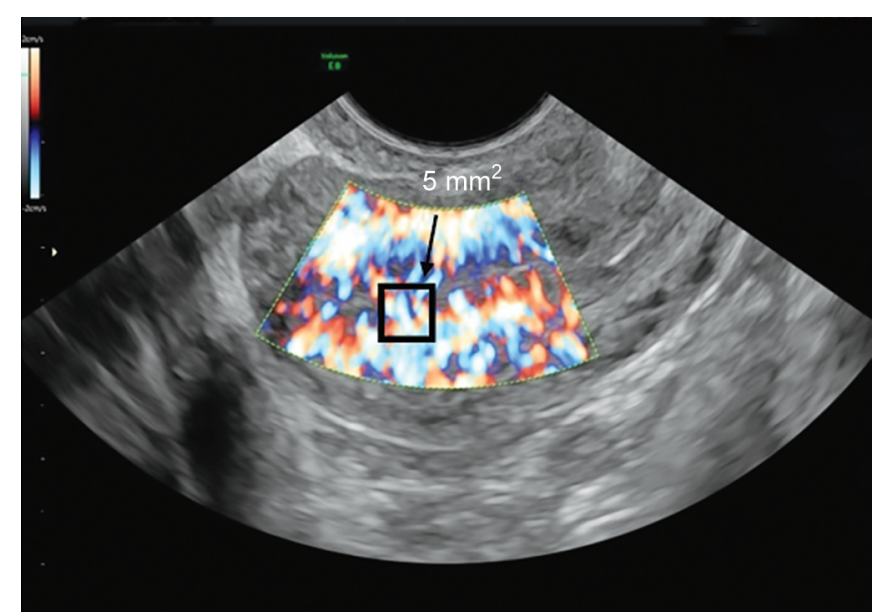

Fig. 4: $5 \mathrm{~mm}^{2}$ area in zone 3 showing vascularity on HD power Doppler

Endometrial thickness of $>6 \mathrm{~mm}$, was taken as a cut-off to start progesterone and plan embryo transfer. If, when the scan is done on day 8-9 of the cycle and the endometrial growth is not documented as compared to the first scan, the dose of estradiol valerate was increased to $8 \mathrm{mg}$. Estrogen priming of the endometrium for a minimum of 12 days and maximum up to 28 days is done before progesterone was started as a final preparation for embryo transfer. Preparation of the endometrium for embryo transfer is done with natural micronized progesterone gel $8 \%$ twice a day and subcutaneous administration of aqueous progesterone $25 \mathrm{mg}$; for all the patients undergoing frozen embryo transfer. Estrogen is continued in the dose; that was given before starting progesterone.

Day 5 embryo transfer was done in all the cases. The cryopreserved embryos were thawed on the day of the embryo transfer. Two Grade 1; blastocyst were transferred in all patients. Luteal phase was supported with progesterone, estradiol valerate and aspirin in the same dose as before the transfer to support implantation after the embryo transfer.

Serum $\beta$-hCG level estimation was done after 14 days of the embryo transfer. Serum $\beta$-hCG level more than $10 \mathrm{mlU} / \mathrm{ml}$ was indicative of a positive pregnancy test which was the primary desired result. If the pregnancy test was positive, same luteal support was continued till 12 weeks of the pregnancy. Transvaginal ultrasound was performed 10 days after positive $\beta$-hCG to diagnose a clinical pregnancy. Presence of gestational sac in the endometrial cavity was considered as ultrasound evidence of pregnancy.

Pregnancy was considered only biochemical when no evidence of an intrauterine or extrauterine gestational sac on transvaginal ultrasonography with beta human chorionic gonadotropi ( $\beta$-hCG) levels $>10 \mathrm{miu} / \mathrm{ml}$ but not increasing to more than double in 72 hours. Failure of the appearance of the yolk sac at $20 \mathrm{~mm}$ gestational sac or no foetal pole at $25 \mathrm{~mm}$ of gestational sac is considered a failed pregnancy. ${ }^{15}$ The gestational sac does not grow at the normal rate of $1-1.3 \mathrm{~mm} /$ day $^{16}$ or $4 \mathrm{~mm} /$ week was also considered as failure of pregnancy. Weekly follow-up was done up to eight weeks to confirm ongoing pregnancy. The miscarriage group included patients with biochemical pregnancy and clinical miscarriage.

\section{Results}

The current study included 226 patients who underwent Frozen-embryo transfer. The pre-procedure assessment included assessment of endometrial thickness, morphology and endometrial vascularity on the day progesterone was started as a final endometrial preparation for frozen embryo transfer. Progesterone was started when the endometrial thickness was at least $6 \mathrm{~mm}$ when Estradiol valerate was given for minimum 12 days to maximum 28 days.

The study showed overall pregnancy rate of $42.92 \%(97 / 226)$ based on $\beta$-hCG levels and included 14 miscarriages (14.43\%) out of the 97 positive pregnancies. The average age of the patients in the pregnant group was 32.3 years and that in the nonpregnant group was 31.9 years and $\mathrm{BMI}$ in both the groups was between 22 and 30.

For evaluation of the results and the aim to find the most favorable endometrial thickness and vascularity for conception and to minimize miscarriage rates, the patients were divided primarily based on the result of the frozen transfer cycle-positive or negative $\beta$-hCG results. The results for both the groups were analyzed. The results were compared for both the groups for different endometrial thickness range and for different zones of vascularity.

Assessing the endometrial thickness distribution (Table 1), $17 / 226(7.52 \%)$ patients had endometrial thickness of $\leq 7 \mathrm{~mm}$, 103/226 (45.57\%) had endometrial thickness between 7 and $9 \mathrm{~mm}$, 70/226 (30.97\%) patients had endometrial thickness between 9 and $11 \mathrm{~mm}, 28 / 226(12,38 \%)$ patients has endometrial thickness of between 11 and $13 \mathrm{~mm}$ and $8 / 226$ (3.53\%) patients had endometrial thickness of between 13 and $15 \mathrm{~mm}$.

Of all the patients (Fig. 5); 4/17 (23.25\%) patients with endometrial thickness $<7 \mathrm{~mm}, 49 / 103$ (47.57\%) patients with endometrial thickness of 7-9 mm, 27/70 (38.57\%) patients with endometrial thickness of $9-11 \mathrm{~mm}, 13 / 28$ (46.42\%) patients between endometrial thickness of $11-13 \mathrm{~mm}$ and $5 / 8(50 \%)$ patients with endometrial thickness of $13-15 \mathrm{~mm}$ got positive 


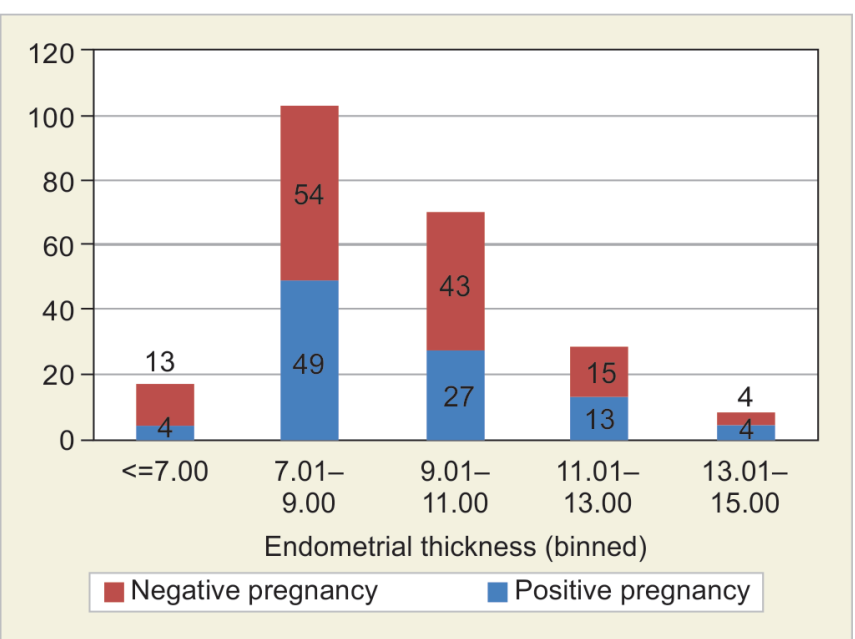

Fig. 5: Endometrial thickness and the pregnancy outcome

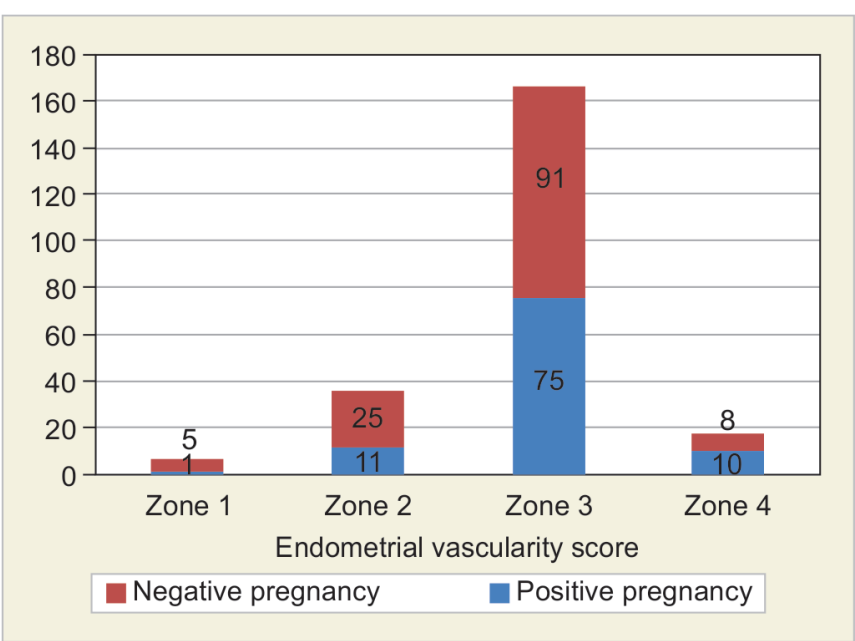

Fig. 6: Endometrial vascularity zone and pregnancy outcomes

Table 1: Endometrial thickness (binned) and pregnancy result-cross tabulation

\begin{tabular}{lccccc}
\hline & & Positive pregnancy & Negative pregnancy & Total patients & Pregnancy rate \\
\hline Endometrial thickness & $\leq 7.00$ & 4 & 13 & 17 & $23.52 \%$ \\
(Binned) & $7.01-9.00$ & 49 & 54 & 103 & $47.57 \%$ \\
& $9.01-11.00$ & 27 & 43 & 70 & $38.57 \%$ \\
& $11.01-13.00$ & 13 & 15 & 28 & $46.42 \%$ \\
Total & $13.01-15.00$ & 4 & 4 & 226 & $50 \%$ \\
\hline
\end{tabular}

Table 2: Endometrial vascularity score and pregnancy result-cross tabulation

\begin{tabular}{lccccc}
\hline & & Positive pregnancy & Negative pregnancy & Total patients & Pregnancy rate \\
\hline Endometrial vascularity & Zone 1 & 1 & 5 & 6 & $16.66 \%$ \\
score & Zone 2 & 11 & 25 & 36 & $30.55 \%$ \\
& Zone 3 & 75 & 91 & 166 & $45.18 \%$ \\
& Zone 4 & 10 & 8 & 18 & $55.55 \%$ \\
Total & & 97 & 129 & 226 & \\
\hline
\end{tabular}

Table 3: Endometrial thickness and abortion

\begin{tabular}{llcccc}
\hline & & No abortion & Abortion & Total pregnancies & Abortion rate \\
\hline Endometrial & $\leq 7.00$ & 5 & 0 & 5 & 0 \\
thickness (Binned) & $7.01-9.00$ & 42 & 9 & 51 & $17.64 \%$ \\
& $9.01-11.00$ & 20 & 4 & 24 & $16.66 \%$ \\
& $11.01-13.00$ & 12 & 1 & 13 & $7.69 \%$ \\
Total & $13.01-15.00$ & 4 & 0 & 4 & 0 \\
\hline
\end{tabular}

pregnancy test. Though the conception rates were significantly lower when endometrial thickness was $\leq 7 \mathrm{~mm}$, for the rest of the endometrial thicknesses the conception rates were not significantly different. Although the conception rates were significantly higher when endometrial thickness was $13-15 \mathrm{~mm}$.

Assessing patient distribution for endometrial vascularity, $6 / 226$ (2.65\%) patients had vascularity in zone 1, 36/226 (15.92\%) had vascularity in zone $2,166 / 226(73.45 \%)$ patients vascularity in zone $3,18 / 226$ (7.96\%) patients had vascularity in zone 4 (Table 2).

Of all the patients (Fig. 6) 1/6 (16.66\%) patients with endometrial vascularity in zone 1, 11/36 (30.55\%) patients with vascularity in zone $2,77 / 166$ (45.18\%) patients with endometrial vascularity in zone 3 and 10/18 (55.55\%) patients with endometrial vascularity in zone 4 got positive pregnancy test.

The findings suggest that the conception rate consistently increased as the vascularity penetrated deeper in the endometrium.

Evaluating the secondary aim of the study, the abortion rate in relation to the endometrial thickness. For endometrial thickness of $<7 \mathrm{~mm}$, abortion rate were calculated as $0 \%$, for endometrial thickness $7-9 \mathrm{~mm}$, abortion rate was $17.64 \%$, for $9-11 \mathrm{~mm}$, it was $16.66 \%$, $7.69 \%$ for endometrial thickness of $11-13 \mathrm{~mm}$ and again zero for endometrial thickness of $13-15 \mathrm{~mm}$ (Table 3). No definite pattern 
Impact of Intraendometrial Vascularity

Table 4: Endometrial vascularity zone and abortion rates

\begin{tabular}{llccrr}
\hline & & No abortion & Abortion & Total pregnancy & Abortion rate \\
\hline \multirow{2}{*}{$\begin{array}{l}\text { Endometrial vascularity } \\
\text { zone }\end{array}$} & Zone 1 & 3 & 0 & 3 & 0 \\
& Zone 2 & 13 & 6 & 19 & $31.57 \%$ \\
& Zone 3 & 59 & 8 & 67 & $11.94 \%$ \\
Total & Zone 4 & 8 & 0 & 8 & 0 \\
\hline
\end{tabular}

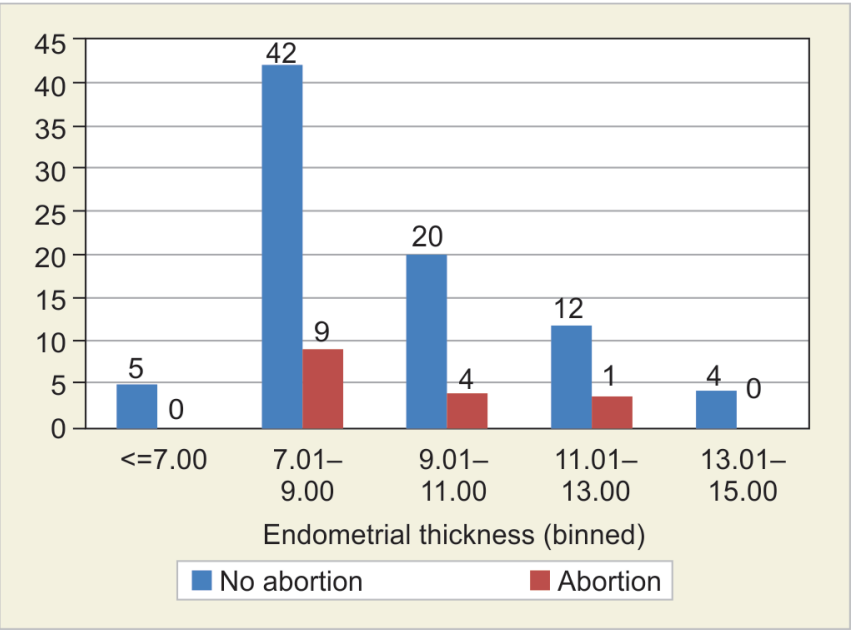

Fig.7: Endometrial thickness and miscarriage rates

of abortion rates were documented with increasing endometrial thickness (Fig. 7). This indicates that the abortion rate cannot be related to the endometrial thickness in frozen embryo transfer cycles.

Evaluating the abortion rates for vascularity in different endometrial zones (Table 4). The abortion rates were (8/67)14.28\% and no abortions were noted when the endometrial vascularity was in zone 3 and zone 4 respectively. Whereas when the vascularity was in zone 2, miscarriage rate was (6/19) $31.57 \%$. Though $(0 / 3)$ abortions were documented when the vascularity was seen in zone 1 only, this was probably too small a number to endorse low abortion rate in this case (Fig. 8).

\section{Discussion}

Implantation is a complex process involving several biochemical and molecular processes. Angiogenesis plays an important role in female reproductive processes such as development of dominant follicle, formation of a corpus luteum, growth of endometrium and implantation. ${ }^{17-19}$ Endometrial angiogenesis is thought to be crucial to endometrial receptivity, but the studies have shown controversial results. The only parameter that has been fairly consistently been shown to be a reliable marker of endometrial receptivity is endometrial thickness.

We preferred to do the endometrial thickness and vascularity studies, on the day when we planned to start progesterone after assessing endometrial thickness, morphology, and vascularity by power Doppler, to maintain the consistency about the time of assessment. There is a decrease in endometrial thickness and vascularity immediately after starting progesterone due to initial compacting effect of progesterone on the endometrium and slow gradual building up of progesterone level to improve the endometrial circulation by decreasing uterine artery resistance

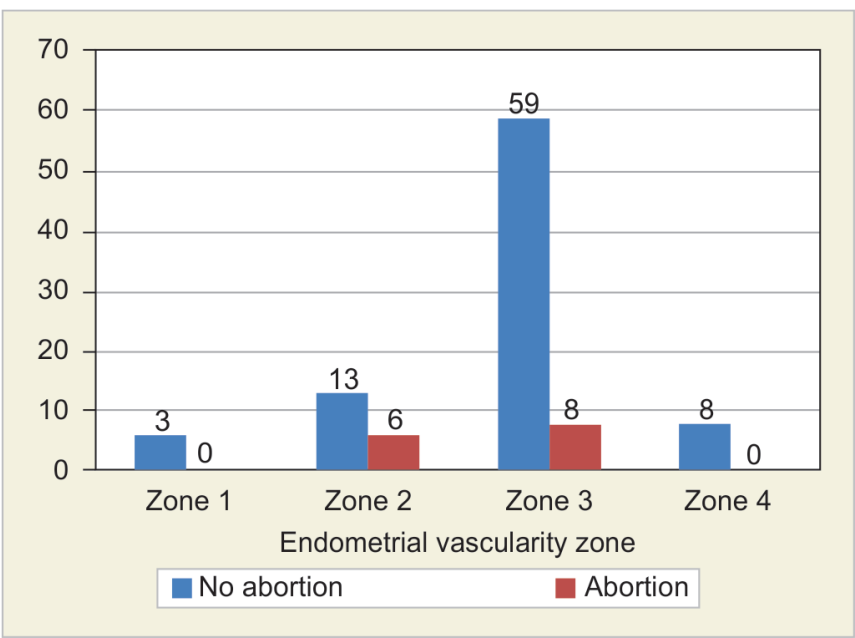

Fig. 8: Endometrial vascularity and miscarriage rate

due to smooth muscle relaxation. ${ }^{20}$ This is the need of physiology also because relatively low oxygen tension is present around the blastocyst during the time of implantation. ${ }^{21}$

Yaman et al. demonstrated in their study that there was no significant difference in the conception and abortion rates in FET cycles, between endometrial thickness of 7-13 mm. ${ }^{22}$ This can be related to our findings too. Several other studies have also shown that endometrial thickness and pattern have low positive predictive value and specificity in the prediction of IVF outcome. ${ }^{3,23-25}$ When the thickness measured by ultrasound is $<7 \mathrm{~mm}$, the functional layer is thin or absent, and the implanting embryo would be much closer to the spiral arteries and the higher vascularity and oxygen concentrations of the basal endometrium. The high oxygen concentration near the basal layer could be detrimental compared with the low oxygen tension of the surface endometrium..$^{26,27,38}$ Singh et al. ${ }^{5}$ reported lower cut-off of $5.8 \mathrm{~mm}$ of endometrial thickness for implantation, with the most favorable thickness of $8-10 \mathrm{~mm}$. Sundström ${ }^{25}$ also reported a successful pregnancy with an endometrial thickness as little as $4 \mathrm{~mm}$. According to these and many other studies therefore it is difficult to establish a definite relationship and the conception rates. ${ }^{28-30}$ These results are in agreement with the results of our present study. Also, lower conception rate was documented with endometrial thickness more than $14 \mathrm{~mm}$, but up to $14 \mathrm{~mm}$ of thickness no significant change in conception rate was found. ${ }^{31}$

When relating the conception rates to endometrial vascularity before starting progesterone as final endometrial preparation in FET cycles in our studies, we have found significantly better conception rates for better endometrial vascularity in terms of vascular penetration (zone of vascularity). This also correlates with our previous study relating endometrial vascularity to conception rates in ovum donation, fresh embryo transfer cycles, when endometrial vascularity was assessed on the day of trigger. $^{32}$ 
Considering and comparing our results with other workers, Mojgan et al. ${ }^{33}$ found that women who had a more favorable cycle showed a higher endometrial vascular flow rate as against the non-pregnant ones in IUI cycles. Riad et al. ${ }^{34}$ also reported that the presence of subendometrial flow is associated with successful IUI in women under stimulated cycles undergoing IUI. Wu et al. ${ }^{35}$ also demonstrated in 54 patients that subendometrial VFI on the day of hCG was significantly higher in the pregnant group and was superior to endometrial volume, subendometrial $\mathrm{VI}$ and $\mathrm{Fl}$ in predicting the outcome. This indicates that not just the subjective assessment of more vascular penetration, even quantitative assessment of the endometrial vascularity proved that better conception rates can be achieved when endometrium was well vascularized on the day of trigger in IVF-fresh transfer cycles.

Wang et al. ${ }^{4}$ found in prospective study of 182 patients undergoing IVF-ET, that patients with detected endometrial blood flow had a higher pregnancy and implantation rate and suggested that blood supply and distribution of the endometrium had a strong association with the possibility for embryo implantation and development, indicating the value of detecting the existence of endometrial blood flow in predicting IVF-ET outcomes. They also found a significant increasing multiple pregnancy rate in those who had better endometrial blood flow. We in our study also found that deeper the penetration of vascularity in endometrium, better is the positive pregnancy outcome. Nygren et al. ${ }^{36}$ have also suggested positive correlation of the endometrial and subendometrial blood flows to the outcome of IVF/ET.

In a recent paper, $\mathrm{Ng}$ et al. ${ }^{37}$ discuss the relationship of endometrial blood flow between those with a thin (less than or equal to $8 \mathrm{~mm}$ ) endometrium and in those with a low volume (less than or equal to $2.5 \mathrm{ml}$ ) endometrium. Endometrial and subendometrial vascularity measured by 3D power Doppler ultrasound was significantly lower ( $p$ value less than or equal to 0.003 ) in patients with a low volume endometrium, but not in those with a thin endometrium. Therefore, according to this study, considering the vascular parameters along with endometrial thickness provides better positive pregnancy outcomes then only when the endometrial morphological parameters and its thickness are taken into account.

Dechaud et al. ${ }^{39}$ considered that, the endometrial pattern, thickness and end-diastolic blood flow were shown to be the most effective combination for the evaluation of uterine receptivity. Divya sardana et al. ${ }^{40}$ also suggested that a combination of endometrial thickness and Doppler analysis of the endometrial blood flow was a simple and effective tool to improve the outcome of IVF/ET. But we have not found any correlation between the endometrial thickness and conception rates when the endometrial thickness was between 7 and $13 \mathrm{~mm}$.

Though Ng et al. ${ }^{41}$ did not demonstrate a significant difference in vascularity parameters of the endometrium and positive pregnancy outcome, when endometrial vascularity assessment was done a day after the LH surge started in natural cycle or clomiphene citrate stimulated FET cycles. This may correlate with the known decreased vascularity of the endometrium soon after the start of LH surge. Raine-Fenning et al. ${ }^{42}$ proposed that the degree of change in endometrial perfusion from the late follicular phase through to the early luteal phase was a more important determinant of endometrial receptivity. Thus indicating that the dynamic changes in the endometrial vascularity are more relevant to the possibility of conception and serial assessment of the endometrial vascularity may better predict and relate to conception rates.

About $10-15 \%$ of clinically recognised pregnancies end in spontaneous miscarriage. Though $50 \%$ of the first trimester spontaneous miscarriage may have an abnormal karyotype, ${ }^{43}$ in the rest it may also be due to abnormal uterine or endometrial blood flow indices. ${ }^{44}$ Measuring the endometrial blood flow indices in IVF and FET treatment, even before the time of implantation, may open up a possibility to start therapeutic measures to reduce the miscarriage in these patients to improve endometrial flow. In one of our previous studies, we have also found significantly lower abortion rates in IUI cycles when the conception had occurred with vascularity in zone 3 and zone 4 of the endometrium on the day of ovulation trigger. ${ }^{45}$ Chien et al. stated that, when pregnancy is achieved in absence of endometrial and subendometrial flow on the day of embryo transfer, more than half of these pregnancies will finish as spontaneous miscarriage. ${ }^{46}$

In one of our other studies; patients with endometrial vascularity in zones 3 and $4, \mathrm{RI}<0.6$, had a high conception rate and low miscarriage rate but with endometrial volume of $<1.5 \mathrm{ml}$, not a single pregnancy was noted. If the endometrial FI before the trigger for ovulation is between 37 and 41 and endometrial VFI was between 25 and 27, not only there are significantly higher chances of leading to conception, but these values also carry a high chance of ongoing pregnancy. ${ }^{47}$ In another study by $\mathrm{Ng}$ et al., a multiple logistic regression analysis, only endometrial VI was significantly associated with the chance of live birth with an odds ratio (OR) of 1.384 [95\% confidence interval $(\mathrm{Cl}) 1.025-1.869, p=$ 0.034]. In the prediction of live birth, in FET cycles, the area under the receiver operating characteristic (ROC) curve for endometrial $\mathrm{VI}$ was 0.64 and the odds ratio (OR) was $3.2(95 \% \mathrm{Cl}$ : 1.5-6.8) with a cut-off of $>0.306 .^{13}$ This study indicates better endometrial perfusion indicates higher chances of live birth and is consistent with the results of our study.

\section{Conclusion}

This study indicates that zone 3 (intra-endometrial) vascularity is an important parameter when evaluating endometrial receptivity and can be positively correlated to the conception rates and negatively correlated with the miscarriage rates. We recommend; therefore, to evaluate the endometrium before FET by Doppler and decide on starting progesterone for frozen embryo transfer only if intra-endometrial vascularity is seen.

\section{References}

1. Coulam CB, Bustillo M, Soenksen DM, et al. Ultrasonographic predictors of implantation after assisted reproduction. Fertil Steril 1994;62:1004-1010. DOI: 10.1016/s0015-0282(16)57065-4

2. DomínguezF, Remohí J, Pellicer A, etal. Human endometrialreceptivity: a genomic approach. Reprod Biomed Online 2003;6:332-338. DOI: 10.1016/s1472-6483(10)61853-6

3. Turnbull LW, Lesny P, Killick SR. Assessment of uterine receptivity prior to embryo transfer: a review of currently available imaging modalities. Hum Reprod Update 1995;1:505-514. DOI: 10.1093/humupd/1.5.505

4. Wang L, Qiao J, Li R, et al. Role of endometrial blood flow assessment with color Doppler energy in predicting pregnancy outcome of IVF-ET cycles. Reprod Biol Endocrinol 2010;8:122-128. DOI: 10.1186/1477-7827-8-122

5. Singh N, Bahadur A, Mittal S, et al. Predictive value of endometrial thickness, pattern and sub-endonetrial blood flows on the day of hCG by 2D Doppler in in-vitro fertilization cycles: a prospective 
clinical study from a tertiary care unit. J Hum Reprod Sci 2011;4:29-33. DOI: 10.4103/0974-1208.82357

6. Horcajadas JA, Pellicer A, Simon C. Wide genomic analysis of human endometrial receptivity: new times, new opportunities. Hum Reprod Update 2007;13:77-86. DOI: 10.1093/humupd/dml046

7. $\mathrm{Ng} \mathrm{EH}$, Chan CC, Tang OS, et al. The role of endometrial and subendometrial blood flows measured by three dimensional power Doppler ultrasound in the prediction of pregnancy during IVF treatment. Hum Reprod 2006;21:164-170. DOI: 10.1093/humrep/dei277

8. Bakos O, Lundkvist O, Bergh T. Transvaginal sonographic evaluation of endometrial growth and texture in spontaneous ovulatory cycles-a descriptive study. Hum Reprod 1993;8:799-806. DOI: 10.1093/oxfordjournals.humrep.a138145

9. Smith B, Porter R, Ahuja K, et al. Ultrasonic assessment of endometrial changes in stimulated cycles in an in vitro fertilization and embryo transfer program. J In Vitro Fert Embryo Transf 1984;1:233-238. DOI: 10.1007/BF01131622

10. Applebaum M. The steel or teflon endometrium-ultrasound visualization of endometrial vascularity in IVF patients and outcome. Presented at the third World Congress of ultrasound inobstetricsand gyneacology. Ultrasound Obstet Gynecol 1993;3(Suppl. 2):10.

11. Yang JH, Wu MY, Chen CD, et al. Association of endometrial blood flow as determined by a modified color Doppler technique with subsequent outcome of in-vitro fertilization. Hum Reprod 1999;14:1606-1610. DOI: 10.1093/humrep/14.6.1606

12. Zaidi J, Pittrof R, Shaker A, et al. Assessment of uterine artery blood flow on the day of human chorionic gonadotrophin administration by transvaginal color Doppler ultrasound in an in vitro fertilization program. Fertil Steril 1996;65(2):377-381. DOI: 10.1016/s00150282(16)58103-5

13. Ng EHY, Wai Chan CC, Tang OS, et al. Endometrial and subendometrial vascularity is higher in pregnant patients with livebirth following ART than in those who suffer a miscarriage. Hum Reprod 2007;22(4):1134-1141. DOI: 10.1093/humrep/del458

14. Kupesic S, Bekavac I, Bjelos D, et al. Assessment of endometrial receptivity by transvaginal color Doppler and 3D power Doppler ultrasonography in patients undergoing in vitro fertilization procedures. J Ultrasound Med 2001;20:125-134. DOI: 10.7863/ jum.2001.20.2.125

15. Doubilet PM, Benson CB, Bourne T, et al. Diagnostic criteria for nonviable pregnancy early in the first trimester. $\mathrm{N}$ Engl J Med 2013;369(15):1443-1451. DOI: 10.1056/NEJMra1302417

16. Nyberg DA, Mack LA, Laing FC, et al. Distinguishing normal from abnormal gestational sac growth in early pregnancy. J Ultrasound Med 1987;6(1):23-27. DOI: 10.7863/jum.1987.6.1.23

17. Demir R, Kayisli UA, Cayli S, et al. Sequential steps during vasculogenesis and angiogenesis in the very early human placenta. Placenta 2006;27:535-539. DOI: 10.1016/j.placenta.2005.05.011

18. Nardo LG. Vascular endothelial growth factor expression in the endometrium during the menstrual cycle, implantation window and early pregnancy. Curr Opin ObstetrGynecol 2005;17:419-423. DOI: 10.1097/01.gco.0000175362.12470.e0

19. Sherer DM, Abulafia O. Angiogenesis during implantation, and placental and early embryonic development. Placenta 2001;22:1-13. DOI: $10.1053 /$ plac. 2000.0588

20. Sharkey AM, Day K, McPherson A, et al. Vascular endothelial growth factor expression in human endometrium is regulated by hypoxia. J Clin Endocrinol Metab 2000;85:402-409. DOI: 10.1210/jcem.85.1.6229

21. Gardner DK. The impact ofphysiological oxygen during culture, and vitrification for cryopreservation, on the outcome of extended culture in human IVF. Reprod Biomed Online 2016;32:137-141. DOI: 10.1016/j.rbmo.2015.11.008

22. Yaman C, Ebner T, Jesacher K, et al. Sonographic measurement of endometrium thickness as a predictive value for pregnancy through IVF. Ultraschall Med 2002;23:256-259. DOI: 10.1055/s-2002-34053

23. Friedler S, Schenker JG, Herman A, et al. The role of ultrasonography in the evaluation of endometrial receptivity following assisted reproductive treatments: a critical review. Hum Reprod Update 1996;2(4):323-335. DOI: 10.1093/humupd/2.4.323

24. Remohí J, Ardiles G, García-Velasco JA, et al. Endometrial thickness and serum oestradiol concentrations as predictors of outcome in oocyte donation. Hum Reprod 1997;12(10):2271-2276. DOI: $10.1093 /$ humrep/12.10.2271

25. Sundström P. Establishment of a successful pregnancy following in-vitro fertilization with an endometrial thickness of no more than $4 \mathrm{~mm}$. Hum Reprod 1998;13(6):1550-1552. DOI: 10.1093/humrep/13.6.1550

26. Weissman A, Gotlieb L, Casper RF. The detrimental effect of increased endometrial thickness on implantation and pregnancy rates and outcome in an in vitro fertilization program. Fertil Steril 1999;71:147-149. DOI: 10.1016/s0015-0282(98)00413-0

27. Zhao J, Zhang Q, Li Y. The effect of endometrial thickness and pattern measured by ultrasonography on pregnancy outcomes during IVF-ET cycles. Reprod Biol Endocrinol 2012;10:100. DOI: 10.1186/1477-7827-10-100

28. Rieger L, Honig A, Sutterlin M, et al. Antigen-presenting cells in human endometrium during the menstrual cycle compared to early pregnancy. J Soc Gynecol Investig 2004;11:488-493. DOI: 10.1016/j. jsgi.2004.05.007

29. Kolibianakis EM, Zikopoulos KA, Fatemi HM, et al. Endometrial thickness cannot predict ongoing pregnancy achievement in cycles stimulated with clomiphene citrate for intrauterine insemination. Reprod Biomed Online 2004;8:115-118. DOI: 10.1016/s1472-6483(10)60505-6

30. Vlaisavljevic V, Reljic M, Gavric-Lovrec V, et al. Subendometrial contractility is not predictive for in vitro fertilization (IVF) outcome. Ultrasound Obstet Gynecol 2001;17:239-244. DOI: 10.1046/j.1469-0 705.2001.00316.x

31. Dietterich C, Check JH, Choe JK, et al. Increased endometrial thickness on the day of human chorionic gonadotropin injection does not adversely affect pregnancy or implantation rates following in vitro fertilization-embryo transfer. Fertil Steril 2002;77:781-786. DOI: 10.1016/S0015-0282(01)01727-7

32. Nagori $C$, Panchal S. Endometrial vascularity: its relation to implantation rates. Int J Infertility Fetal Med 2012;3(2):48-50. DOI: 10.5005/jp-journals-10016-1040

33. Masrour MJ, Shafaie A, Yoonesi L, et al. Evaluating endometrial thickness and vascular ultrasound pattern and pregnancy outcomes in intrauterine insemination cycle. Asian J Pharm Res Health Care 2016;8(S1):24-29. DOI: 10.18311/ajprhc/2016/7718

34. Riad ON, Hak AA. Assessment of endometrial receptivity using Doppler ultrasonography in infertile women undergoing intrauterine insemination. Gynecol Endocrinol 2014;30:70-73. DOI: 10.3109/09513590.2013.859668

35. Wu HM, Chiang $\mathrm{CH}$, Huang $\mathrm{HY}$, et al. Detection of the subendometrial vascularization flow index by three-dimensional ultrasound may be useful for predicting the pregnancy rate for patients undergoing in vitro fertilization-embryo transfer. Fertil Steril 2003;79:507-511. DOI: 10.1016/s0015-0282(02)04698-8

36. Nygren KG, Sullivan E, Zegers-Hochschild F, et al. International Committee for Monitoring Assisted Reproductive Technology (ICMART) world report: assisted reproductive technology. Fertil Steril 2011;95:2209-2222. DOI: 10.1016/j.fertnstert.2011.03.058

37. Ng EH, Yeung WS, Ho PC. Endometrial and subendometrial vascularity are significantly lower in patients with endometrial volume $2.5 \mathrm{ml}$ or less. Reprod Biomed Online 2009;18:262-268. DOI: 10.1016/s1472-6483(10)60264-7

38. Casper RF. It's time to pay attention to the endometrium. Fertil Steril 2011;96(3):519-521. DOI: 10.1016/j.fertnstert.2011.07.1096

39. Dechaud H, Bessueille E, Bousquet PJ, et al. Optimal timing of ultrasonographic and Doppler evaluation of uterine receptivity to implantation. Reprod Biomed Online 2008;16:368-375. DOI: 10.1016/s1472-6483(10)60598-6

40. Sardana D, Upadhyay AJ, K Deepika, et al. Correlation of subendometrial-endometrial blood flow assessment by 
two-dimensional power Doppler with pregnancy outcome in frozen-thawed embryo transfer cycles. J Hum Reprod Sci 2014;7(2):130-135. DOI: 10.4103/0974-1208.138872

41. Ng EHY, Chan CCW, Tang OS, et al. The role of endometrial and subendometrial vascularity measured by three-dimensional power Doppler ultrasound in the prediction of pregnancy during frozen thawed embryo transfer cycles. Hum Reprod 2006;21:1612-1617. DOI: 10.1093/humrep/dei502

42. Raine-Fenning NJ, Campbell BK, Kendall NR, et al. Endometrial and subendometrial perfusion are impaired in women with unexplained subfertility. Hum Reprod 2004;19:2605-2614. DOI: 10.1093/humrep/deh459

43. Hassold TJ. Chromosome abnormalities in human reproductive wastage. Trends Genet 1986;2:105-110. DOI: 10.1016/0168-9525(86)90194-0
44. Aleman A, Althabe F, Belizan J, et al. Bed rest during pregnancy for preventing miscarriage, Cochrane Database Syst Rev 2005;2: CD003576. DOI: 10.1002/14651858.CD003576.pub2

45. Panchal S, Nagori C. Endometrial vascularity: its relation to implantation rates. Int J Infertil Fetal Med 2012;3(2):48-50. DOI: 10.5005/jp-journals-10016-1040.

46. Chien LW, Au HK, Chen PL, et al. Assessment of uterine receptivity by the endometrial-subendometrial blood flow distribution pattern in women undergoing in vitro fertilization embryo transfer. FertilSteril 2002;78(2):245-251. DOI: 10.1016/s00150282(02)03223-5

47. Panchal S, Nagori C, Thaker M. Doppler and 3D power Doppler assessment of the endometrium and its correlation to abortion rates. Donald School J Ultrasound Obstet Gynecol 2020;14(2):150-156. DOI: $10.5005 /$ jp-journals-10009-1638 